\title{
Effect of Equilibrium Contact Angle on Drainage Property at Microperiodic Structure with Staggered Pillar Array
}

\author{
Masaki Yamaguchi, ${ }^{*}$ Tetsuhiro Sakata, and Tsuyoshi Chiba \\ Department of Mechanical Engineering \& Robotics, Graduate School of Science \& Technology, \\ Shinshu University, 3-15-1 Tokida, Ueda, Nagano 386-8567, Japan \\ (Received December 15, 2016; accepted January 20, 2017)
}

Keywords: periodic structure, drainage property, equilibrium contact angle, sliding angle, staggered array, micro-electromechanical systems (MEMS)

The purpose of this research is to clarify the effects of the equilibrium contact angle on the drainage property of water from microperiodic structures. Resin samples with 3 different equilibrium contact angles were fabricated by lithography and the UV nanoimprint technique. To consider the effect of the Cassie-Baxter and Wenzel states on drainage property, four types of microperiodic structures with varying aspect ratios were fabricated. Thus, in total, 12 test pieces, each consisting of a staggered pillar array, were used. We attempted to improve the drainage property by optimizing the microperiodic structure. A tilted plate method was modified to evaluate these microperiodic structures. From the results, the sliding angle and sliding velocity reached $14^{\circ}$ and $171.9 \mathrm{~mm} / \mathrm{s}$, respectively, when a material with a sliding angle of $65^{\circ}$ on a smooth surface was used. A promising result is that the threshold level for hydrophobicity introduced by the microperiodic structures was below $90^{\circ}$ for a water droplet in the Cassie-Baxter state.

\section{Introduction}

Since the explanation of the angle on a rough surface, namely, apparent contact angle, on the basis of the Cassie and Wenzel models ${ }^{(1,2)}$ was first put forward, the periodic structures on solid surfaces have been examined to control the wettability. ${ }^{(3,4)}$ The Cassie regime is applied when dealing with a heterogeneous surface, and is described by the Cassie-Baxter equation. On the other hand, the Wenzel regime describes the homogeneous wetting surface, and the static contact angle on a flat material surface, i.e., the equilibrium contact angle, is given by the Wenzel equation. The relationship between the wettability and the proportion of air trapped on a rough surface was shown to conform to the Cassie-Baxter model of wettability. ${ }^{(5,6)}$ Recently, some industrial applications requiring periodic structures with improved dynamic characteristics for the wettability, such as better drainage property, low adhesion, and reduced drag, have come to the fore. ${ }^{(7,8)}$ In particular, the natural or artificial removal of surface water from an area, i.e., the drainage property, is the most important property. Until today, enormous patterns of the periodic structures have been reported, and a staggered pillar array is one of the better patterns for obtaining outstanding drainage properties. ${ }^{(9)}$ However, the Cassie and Wenzel models do not exhaust all possible wetting regimes; actually, the situation is much more complicated..$^{(10,11)}$

*Corresponding author: e-mail: masakiy@shinshu-u.ac.jp http://dx.doi.org/10.18494/SAM.2017.1561 
The sliding angle of water droplets is a useful indicator of the drainage properties. ${ }^{(12)}$ A tilted plate method is used to determine the sliding angle on a flat surface. ${ }^{(13)}$ In this method, a water droplet is placed on a horizontal plate (substrate), and then the plate is gradually inclined until the water droplet begins to slide. However, the water droplet might be trapped by contact line pinning (pinning effect) when a plate with periodic structures on the surface is used. ${ }^{(14)}$ Thus, this protocol may not be suitable as it is. Research demonstrated that a water droplet was released onto a plate from a defined height because a superhydrophobic surface rolls or bounces the water $\operatorname{droplet}^{(15)}$ The measurement method has not yet been adequately explored.

The static wettability such as apparent contact angle or contact angle hysteresis does not adequately describe the dynamic wettability of periodic structures. It is well recognized in the literature on this topic that the relationship between the static and dynamic wettabilities is not straightforward. ${ }^{(16)}$ One of the reasons for this is that it is difficult to change the absolute value of the equilibrium contact angle optionally for the same periodic structure. The purpose of this study is to clarify the effects of the equilibrium contact angle on the drainage properties evaluated on the basis of the sliding angle and the sliding velocity. The development of micro-electromechanical systems (MEMS) has provided tools for highly precise, reproducible, and scalable methods to fabricate micrometer-sized structures. ${ }^{(17)}$ Thus, we applied this lithography-based approach to produce the desired equilibrium contact angles. Additionally, to achieve superior drainage properties, a staggered pillar array was applied to the periodic structures. The tilted plate method was modified to suit the evaluation of a surface with periodic structures. The apparent contact angles, sliding angles, and sliding velocities were compared for several equilibrium contact angles. Finally, we discuss the properties needed for a solid surface in order to improve the drainage property.

\section{Materials and Methods}

\subsection{Manufacturing and observation of test pieces}

A lithography-based approach $^{(18)}$ was used to fabricate a negative-patterned master mold with four different microperiodic structures on silicon. A photomask with periodic structures was implemented using a chrome mask $(1.0 \pm 0.1 \mu \mathrm{m}$ lines and spaces) on synthetic quartz glass. A 4-inch silicon wafer was coated with a positive-type photoresist (TSMR-V90 27cP, Tokyo Ohka Kogyo Co., Ltd., Japan) using a spin coater (ASC-4000, Actes Inc., Japan). The photoresist was exposed via the pattern on the photomask using a mask aligner (MA6, Suss MicroTec K.K., Japan). The exposed photoresist film was developed using a developer solution (NMD-W 2.38\%, Tokyo Ohka Kogyo Co., Ltd., Japan). Deep reactive ion etching (DRIE) was performed on the 4-inch wafer for 325 cycles ( 1 cycle: $2.5 \mathrm{~s}$ of passivation and $3 \mathrm{~s}$ of gas etching) using a Si DRIE system (MUC-21, Sumitomo Precision Co., Ltd., Japan) and octafluorocyclobutane gas $\left(\mathrm{C}_{4} \mathrm{~F}_{8}\right)$. Finally, the photoresist was removed using a piranha solution, which is a mixture of sulfuric acid $\left(\mathrm{H}_{2} \mathrm{SO}_{4}\right)$ and hydrogen peroxide $\left(\mathrm{H}_{2} \mathrm{O}_{2}\right)$, after which the remaining $\mathrm{SiO}_{2}$ was removed using hydrogen fluoride $(\mathrm{HF})$.

An ultraviolet (UV) nanoimprint technique ${ }^{(19,20)}$ was used to fabricate four different test pieces with microperiodic structures. An UV-curable resin (UVM-23, Soken Chemical \& Engineering Co., Ltd., Japan), with a shrinkage factor of $8.2 \%$, was used as the base material for the fabrication of the test pieces. The storage modulus $\mathrm{E}^{\prime}$ and the loss tangent $\tan \delta$ in the dynamic mechanical analysis were $1.74 \times 10^{9} \mathrm{~Pa}$ and 0.345 , respectively. The coefficients of thermal expansion, $\alpha_{1}$ and 
$\alpha_{2}$, in the thermomechanical analysis were $76 \times 10^{-6} /{ }^{\circ} \mathrm{C}$ and $75 \times 10^{-6} /{ }^{\circ} \mathrm{C}$, respectively. To control the equilibrium contact angle chemically, the UV-curable resin was blended with a fluororesin (SIT08-31-H3, Soken Chemical \& Engineering Co., Ltd., Japan). Positive-patterned test pieces with periodic structures were transferred from the negative-patterned master mold. The spin coater was used in this process. The mixture of resins was dropped onto the master mold, which was then rotated to form a thin-film test piece. A week after fabrication, the equilibrium contact angles of the test pieces were measured. This trial manufacturing process was repeated until the desired values of the equilibrium contact angle were obtained. If the water contact angle is $0^{\circ}<\theta$ $<90^{\circ}$, then the surface is usually called hydrophilic, whereas a surface with a water contact angle of $90^{\circ}<\theta<180^{\circ}$ is usually called hydrophobic. ${ }^{(21)}$ In terms of the equilibrium contact angle, 3 types of test piece were fabricated with low $\left(<90^{\circ}\right)$, middle $\left(>>90^{\circ}\right)$, and high $\left(>90^{\circ}\right)$ equilibrium contact angles, which were named as Types $\mathrm{L}, \mathrm{M}$, and $\mathrm{H}$, respectively.

The surface texture of the test pieces was characterized in terms of the pitch $\tau$, the width of the pillar, $f_{1}$, the spacing between the pillars, $f_{2}$, and the depth of the pillar, $d$, using a noncontact laser confocal microscope ( $1 \mathrm{~nm}$ of resolution for depth, OLS4100, Olympus Co., Japan). Each value was measured 5 times and the mean value was recorded. Additionally, the aspect ratio $f_{2} / f_{1}$ was calculated for each test piece.

\subsection{Measurement of equilibrium contact angles and apparent contact angles}

Prior to evaluating the contact angles, the test pieces were washed for $10 \mathrm{~min}$ in distilled water using an ultrasonic washer (1510J-MT, Emerson, Japan), and the distilled water remaining on the surface was blown off by $\mathrm{N}_{2}$ gas. The test pieces were dried for $30 \mathrm{~min}$ in an incubator (MIR-153, Sanyo Electric Co. Ltd., Japan). Finally, the static electricity of the surface was neutralized by an ionizer (SJ-M400, Keyence Co., Japan) until the surface potentials were confirmed to be less than $-5 \mathrm{~V}$ using a surface electrometer (SK-200, Keyence Co., Japan).

The equilibrium contact angles and apparent contact angles, both of which are static contact angles, were measured using a commercial contact angle analyzer (DM-701, Kyowa Interface Science Co. Ltd., Japan). One microliter of distilled water was dropped from a microsyringe onto the surface of each test piece. The measurements were repeated 5 times using different areas. The highest possible contact angle that can be achieved for a given wetting system is called the advancing contact angle whereas the lowest possible contact angle is called the receding contact angle. ${ }^{(22)}$ The difference between the advancing contact angle and the receding contact angle, namely, the contact angle hysteresis $(\mathrm{CAH})$, was measured by the sessile drop technique.

\subsection{Measurement of sliding angles and sliding velocities}

A contact angle analyzer $\left(1^{\circ}\right.$ of table angular resolution, $12000 \mathrm{fps}$ of time resolution, and 51.5 $(x$-axis $) \times 38.7(z$-axis) $\mu \mathrm{m} /$ pixel of spatial resolution) was used to measure the sliding angles and sliding velocities as shown in Fig. 1. The sphericity of the water droplet is affected by gravity, as shown by the Furmidge equation. ${ }^{(23,24)}$ The tilted plate method was modified to suit the evaluation of the microperiodic structures. The water droplet was given an initial velocity by dropping it from a height $h$ in order to decrease the influence of the pinning effect on the evaluation of the periodic structures. Sliding was judged to have occurred if the distilled water droplet moved more than $20 \mathrm{~mm}$ from its original position. Needles of different gauges $(9,12,15,18$, and 24) were used in order to set the volume of the water droplet as a parameter. 


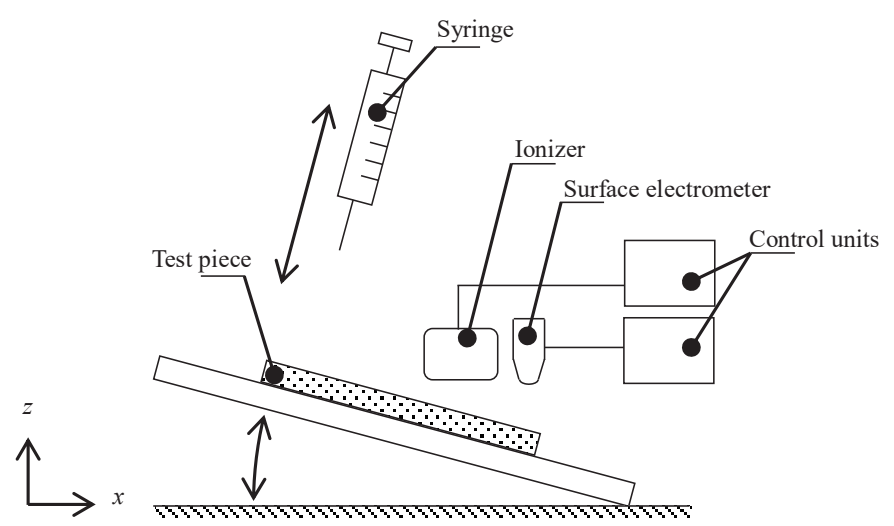

(a)

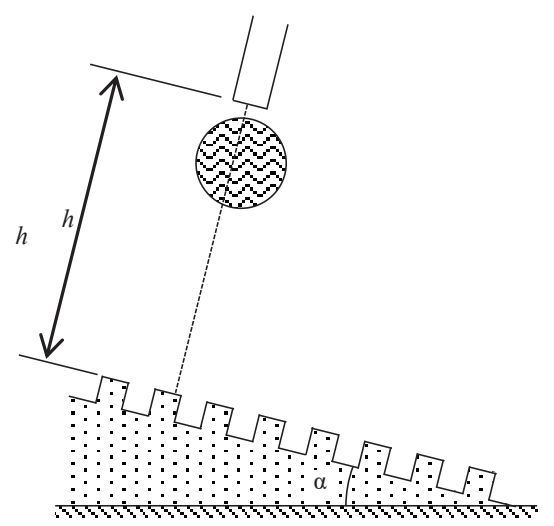

(b)

Fig. 1. Schematic diagram of a commercial contact angle analyzer used for the measurement of the sliding angle and sliding velocity. (a) Contact angle analyzer. (b) Conditions used for measuring sliding angle and sliding velocity ( $\alpha$ : sliding angle).

The sliding velocity was calculated from the speed of the central point between the forwardand backward-facing surfaces of the water droplet $(n=3)$.

\section{Results}

\subsection{Geometry of test pieces}

Four different test pieces with microperiodic structures (area of $30 \times 30 \mathrm{~mm}^{2}$ ), Types A, B, C, and D, were formed on the master mold. These were transferred to UV-curable resin test pieces using the nanoimprint technique. The microperiodic structures were in the form of staggered pillar arrays. The surface textures of the test pieces were examined using a scanning electron microscope (SEM, JSM-6010LA, Jeol Ltd., Japan, Fig. 2). The transfer ratio (test piece/master mold) of the surface textures $\left(\tau, f_{1}, f_{2}\right)$ ranged between $0.88-1.09$ for all types (Table 1$)$. The transfer ratio of the depth $\mathrm{d}$ ranged between $0.90-0.95$ for all types.

\subsection{Equilibrium contact angles and apparent contact angles}

For each type of microperiodic structure, test pieces with 3 different equilibrium contact angles, Types L, M, and H, were fabricated. Thus, 12 different test pieces in total were fabricated $(4 \times 3$ pieces, Table 2$)$. The contact angle on smooth surfaces (i.e., the equilibrium contact angle), $\theta$, ranged between 76.9 (Type $\mathrm{L}$ ) and $103.9^{\circ}$ (Type $\mathrm{H}$ ). The apparent contact angle on surfaces with microperiodic structures, $\theta^{\prime}$, ranged between 90.9 and $147.2^{\circ}$. The maximum increase in static contact angle, $\Delta \theta^{\prime}$, was for Type $\mathrm{C}$ (Fig. 3). Type $\mathrm{L}$ had the minimum increase in contact angle. Type C-M, which has an aspect ratio $f_{2} / f_{1}=2.1$, gave the maximum increase in contact angle at $59.2^{\circ}$ (hydrophobicity). The CAHs on smooth surfaces of Types L, M, and H, were 68.9, 58.9, and $66.7^{\circ}$, respectively. 


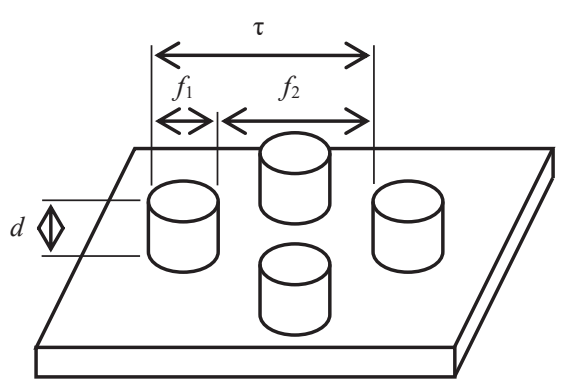

(a)

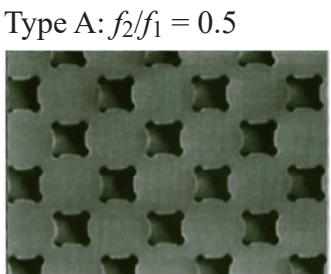

Type C: $f_{2} / f_{1}=2$

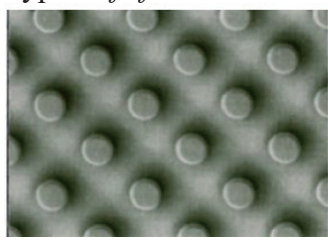

Type B: $f_{2} / f_{1}=1$

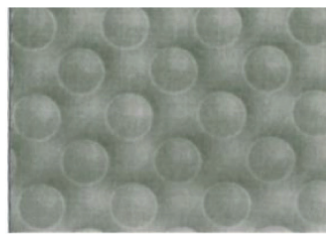

Type D: $f_{2} / f_{1}=5$

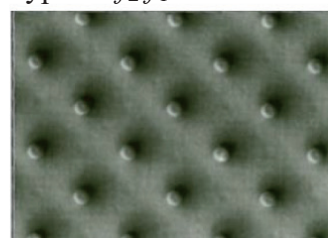

(b)

Fig. 2. (Color online) Parameters of the microperiodic structures and SEM images of the four different types of test pieces. (a) Parameters of a microperiodic structure. (b) Surface textures of test pieces $\left(20 \times 20 \mathrm{~mm}^{2}\right.$ for each sample).

Table 1

Measured results of the sizes of the microperiodic structures (units in $\mu \mathrm{m}$, mean $\pm \mathrm{SD}, n=5$ ).

\begin{tabular}{llcccc}
\hline Type & & $\mathrm{A}$ & $\mathrm{B}$ & $\mathrm{C}$ & $\mathrm{D}$ \\
\hline \multirow{4}{*}{$\tau(\mu \mathrm{m})$} & Designed & 12 & 12 & 12 & 12 \\
& Master mold & $11.85 \pm 0.05$ & $11.88 \pm 0.11$ & $11.91 \pm 0.05$ & $11.91 \pm 0.09$ \\
& Test piece & $11.88 \pm 0.08$ & $11.88 \pm 0.08$ & $11.88 \pm 0.11$ & $11.96 \pm 0.06$ \\
\hline \multirow{3}{*}{$f_{1}(\mu \mathrm{m})$} & Designed & 8 & 6 & 4 & 2 \\
& Master mold & $8.18 \pm 0.33$ & $5.88 \pm 0.06$ & $3.82 \pm 0.05$ & $1.62 \pm 0.05$ \\
& Test piece & $7.86 \pm 0.10$ & $5.56 \pm 0.14$ & $3.59 \pm 0.06$ & $1.42 \pm 0.12$ \\
\hline \multirow{4}{*}{$f_{2}(\mu \mathrm{m})$} & Designed & 4 & 6 & 8 & 10 \\
& Master mold & $3.67 \pm 0.30$ & $6.00 \pm 0.07$ & $8.09 \pm 0.08$ & $10.29 \pm 0.10$ \\
& Test piece & $4.02 \pm 0.05$ & $6.32 \pm 0.18$ & $8.29 \pm 0.06$ & $10.54 \pm 0.17$ \\
\hline \multirow{4}{*}{$f_{2} / f_{1}$} & Designed & 0.5 & 1 & 2 & 5 \\
& Master mold & $0.45 \pm 0.05$ & $1.02 \pm 0.01$ & $2.12 \pm 0.05$ & $6.37 \pm 0.24$ \\
& Test piece & $0.51 \pm 0.01$ & $1.14 \pm 0.06$ & $2.31 \pm 0.03$ & $7.51 \pm 0.73$ \\
\hline \multirow{3}{*}{$d(\mu \mathrm{m})$} & Designed & 6 & 6 & 6 & 6 \\
& Master mold & $6.65 \pm 0.01$ & $6.93 \pm 0.01$ & $6.92 \pm 0.01$ & $6.86 \pm 0.01$ \\
& Test piece & $5.96 \pm 0.04$ & $6.36 \pm 0.01$ & $6.32 \pm 0.02$ & $6.51 \pm 0.05$ \\
\hline
\end{tabular}

Table 2

Measured results of equilibrium contact angle $\theta$ and apparent contact angle $\theta^{\prime}\left(^{\circ}\right)$.

\begin{tabular}{lrrrrrr}
\hline & $\theta$ & \multicolumn{2}{c}{ Type $\mathrm{L}$} & \multicolumn{2}{c}{ Type M } & \multicolumn{2}{c}{ Type H } \\
$f_{2} / f_{1}$ & $\theta$ or $\theta^{\prime}$ & $\Delta \theta^{\prime}$ & $\theta$ or $\theta^{\prime}$ & $\Delta \theta^{\prime}$ & $\theta$ or $\theta^{\prime}$ & $\Delta \theta^{\prime}$ \\
\hline Flat plate & 76.9 & - & 85.8 & - & 103.9 & - \\
A & 105.0 & 28.1 & 108.7 & 22.9 & 112.3 & 8.4 \\
$\mathrm{~B}$ & 106.8 & 29.9 & 132.8 & 47.0 & 137.8 & 33.9 \\
$\mathrm{C}$ & 112.6 & 35.7 & 145.0 & 59.2 & 147.2 & 43.3 \\
$\mathrm{D}$ & 90.9 & 14.0 & 93.4 & 7.6 & 97.3 & -6.6 \\
\hline${ }^{*} \Delta \theta^{\prime}=\theta-\theta^{\prime}$ & & & & & &
\end{tabular}

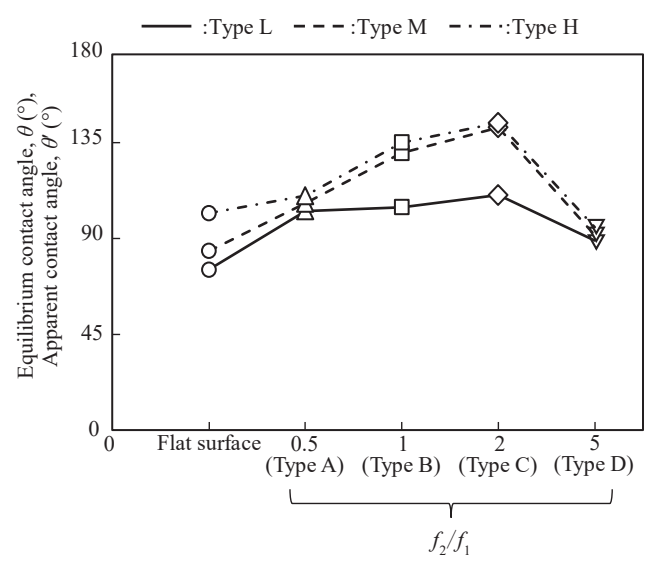

Fig. 3. Measured results of the equilibrium contact angles and apparent contact angles for the 12 different test pieces. 


\subsection{Sliding angles and sliding velocities}

In the tilted plate method, the height $h$ should satisfy the following conditions: (i) the height should be as small as possible, and (ii) the water droplet should be prevented to attach the tilted plate before dropping. In the experiments, a height of $10 \mathrm{~mm}$ was used.

Figure 4 shows the measured results of the relationship between the sliding angle $\alpha$ and the volume of the water droplet, $V$. The volumes were calculated by integrating the side-view area of the water droplet. The minimum values of the sliding angle on the smooth surface of Type $\mathrm{H}$ for volumes of $24.7,38.4$, and $47.0 \mathrm{~mL}$ were 65,45 , and $42^{\circ}$, respectively. The order of the aspect ratio $f_{2} / f_{1}$, in which the sliding angle decreases, was $2,1,5$, and 0.5 , and this tendency was the same for each equilibrium contact angle. The minimum sliding angle on the surfaces with microperiodic structures was $14^{\circ}$ for Type H-C with a water droplet of $24.7 \mu \mathrm{L}$ volume, meaning that the best drainage property occurs for this type under this condition. Furthermore, the sliding angle did not change when the volume of the water droplet was greater than $24.7 \mu \mathrm{L}$. This tendency can be observed for other conditions.

Figure 5 shows the relationship between the sliding angle, $90^{\circ}-\alpha$, and the static contact angles $\theta$ and $\theta^{\prime}$. In other words, this figure shows the relationship between the dynamic and static wettabilities. The correlation coefficient $R$ showed 0.88 except for Type A test pieces $\left(f_{2} / f_{1}=0.5\right)$. Type A had the narrowest grooves of the test pieces and the poorest drainage properties.

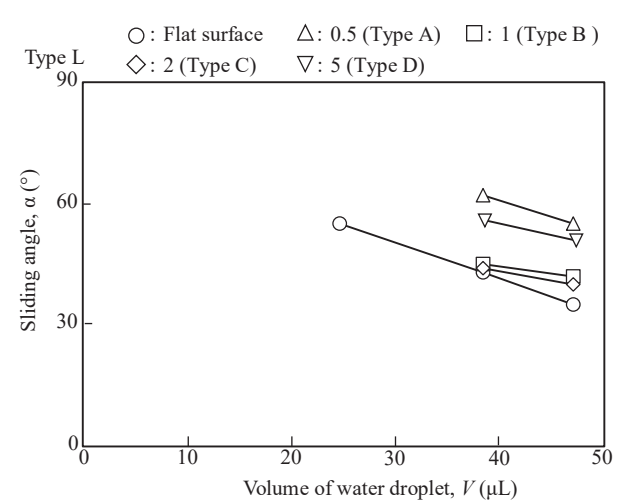

(a)

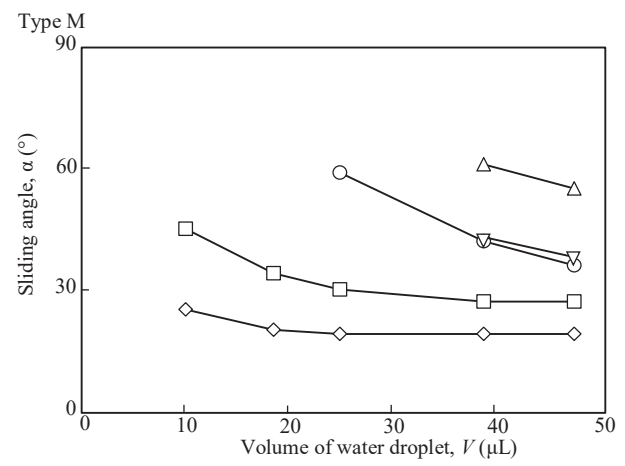

(b)

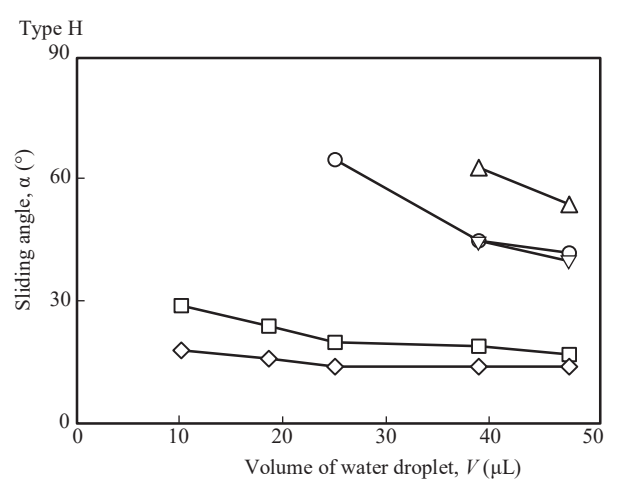

(c)

Fig. 4. Relationship between the sliding angle and the volume of water droplet for each equilibrium contact angle. (a) Type L, (b) Type M, and (c) Type H. 


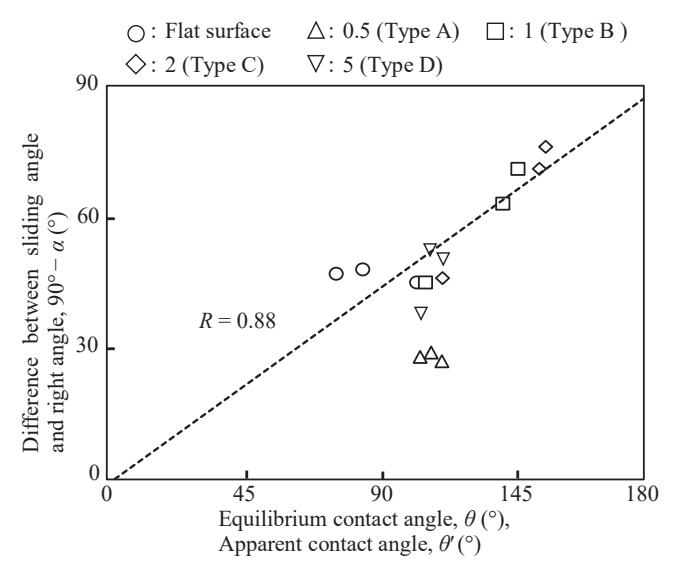

Fig. 5. Relationship between the sliding angle, $90^{\circ}-\alpha$, and the static contact angles $\theta$ and $\theta^{\prime}$ ( $R$ : correlation coefficient).

Figure 6 shows two typical snapshots of water droplets made during the measurements. When both the forward and backward facing sides of the water droplet could be observed visually [Fig. 6(a)], the sliding velocity of the water droplet was measured at the midpoint of the water droplet. On the other hand, when only the forward facing side was observed [Fig. 6(b)], the sliding velocity could not be calculated. All of the observed results for Type A $\left(f_{2} / f_{1}=0.5\right)$ had a slipping trace on the backside and/or the water droplet was trapped on the microperiodic structure, so the sliding velocity could not be measured. The sliding velocity reached $171.9 \mathrm{~mm} / \mathrm{s}$ when the minimum value of the sliding angle ( $14^{\circ}$ by Type $\left.\mathrm{H}-\mathrm{C}\right)$ was obtained.

Figure 7 shows the relationship between the sliding angle, $90^{\circ}-\alpha$, and the sliding velocity of the water droplet, $v$. The results were divided into two groups: Type M (high-speed area) and Type $\mathrm{H}$ (medium-speed area), below and above a sliding velocity of $180 \mathrm{~mm} / \mathrm{s}$. The correlation coefficients for each group had negative values. Furthermore, the results were divided into two groups from viewpoints of $f_{2} / f_{1}=2$ and 1 (high-speed area) in one group and $f_{2} / f_{1}=0.5$ and 5 (lowspeed area) in the other.

\section{Discussion}

In this study, we fabricated resin test pieces with microperiodic structures of $12 \mu \mathrm{m}$ pitch using lithography and the UV nanoimprint technique. The test pieces had different equilibrium contact angles. The transfer ratios of the microperiodic structures ranged between 0.88 and 1.09 , while the equilibrium contact angle was controlled from 76.9 to $103.9^{\circ}$, a ratio between the lowest and the highest of 1.35. This was considered to be a sufficient range for evaluating the drainage properties of these microperiodic structures.

For each equilibrium contact angle, we found that the highest apparent contact angles were for structures with $f_{2} / f_{1}=2$ (Fig. 3). The threshold level of the equilibrium contact angle for hydrophobicity was observed between Types L and M. Bico et al. suggested that the Cassie-Baxter state has a threshold level of the equilibrium contact angle, which was determined by the material. ${ }^{(6,25,26)}$ The optimum surface roughness for hydrophobicity can be realized when 


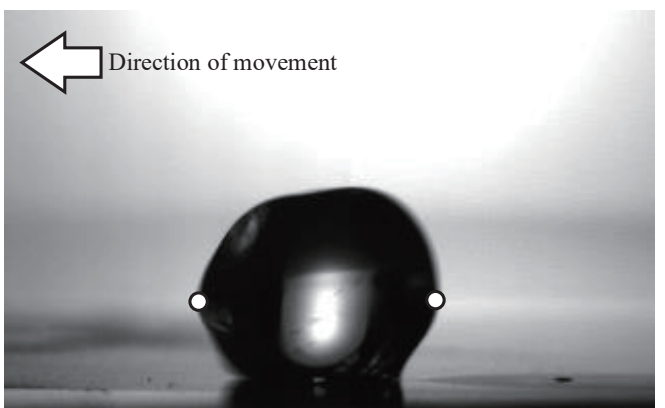

(a)

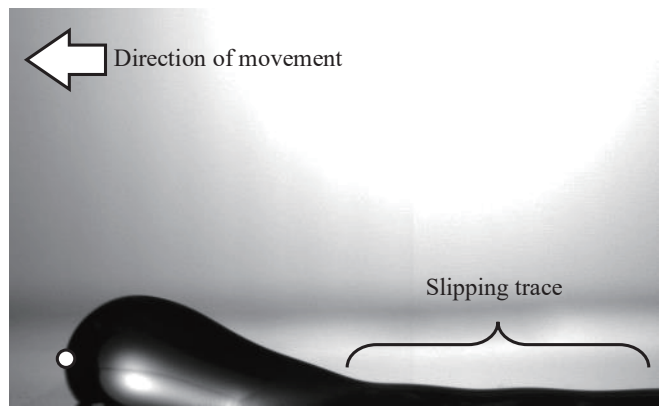

(b)

Fig. 6. Two typical snap shots when the sliding velocities were measured ( 0 : forward- and backward-facing surfaces of water droplet). (a) Type M-B $\left(V=40 \mu \mathrm{L}, \alpha=27^{\circ}\right)$ and (b) Type L-A $\left(V=40 \mu \mathrm{L}, \alpha=62^{\circ}\right)$.

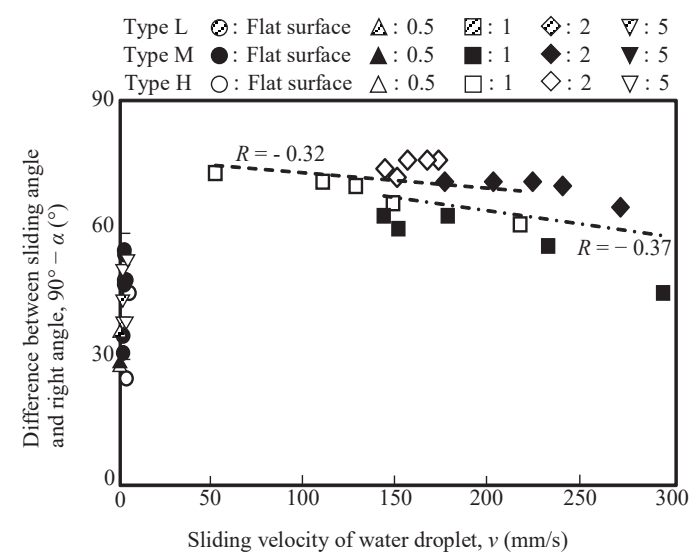

Fig. 7. Relationship between the sliding angle, $90^{\circ}-\alpha$, and the sliding velocity of water droplet ( $R$ : correlation coefficient, 0.5: Type A, 1: Type B, 2: Type C, 5: Type D).

the wetting state is "almost" entirely in the Cassie-Baxter state. Then, the optimum surface roughness is decided by the state of the grooves whether they are filled only with air. Additionally, this phenomenon is affected by not only material but also gravity. ${ }^{(27)}$ Thus, when the water drop is in the Cassie-Baxter state, the threshold level for hydrophobicity of the microperiodic structures may fall critically between Types $\mathrm{L}\left(76.9^{\circ}\right)$ and $\mathrm{M}\left(85.5^{\circ}\right)$, i.e., less than $90^{\circ}$ for these experimental conditions.

The sliding angles gradually decreased in proportion to the volume of the water droplet (Fig. 4). These results agreed well with those in previous reports. ${ }^{(11,28,29)}$ The main reason for this phenomenon is that the grooves are filled with water. That is, the water drop was in the Wenzel state. It was considered that the smallest volume, $38.4 \mathrm{~mL}$ in this case, should be used in the evaluation of the sliding angle, in order to decrease the effect of gravity. The sliding angles for test pieces with $f_{2} / f_{1}=0.5$ were greater than those for flat surfaces. Thus, except for the results of $f_{2} /$ $f_{1}=0.5$, the sliding angles were proportional to the static contact angles (Fig. 5). In other words, if the water droplet is positioned in the Cassie-Baxter state, the static wettability correlates with the dynamic drainage properties. 
In the relationship between the sliding angle, $90^{\circ}-\alpha$, and the sliding velocity of the water droplet, $v$, the correlation coefficients showed negative values $(R=-0.32$ and -0.37 , Fig. 7). The vertical $(x)$ axis can be interpreted as the kinetic friction, including viscus resistance, because the sliding angle is equivalent to the work that is necessary for a droplet to slide down a surface. The larger value along the $x$-axis indicates the smaller sliding angle, and thus the smaller kinetic friction. This phenomenon agreed well with the gravitational acceleration introduced when the tilted plate was angled. It was concluded that the sliding angle is the most useful index for the evaluation of drainage properties, although other conditions such as the volume of the water droplet and the sliding velocity should be set appropriately.

\section{Conclusions}

The effects of the equilibrium contact angle on the drainage properties of microperiodic structures were clarified by fabricating samples with different equilibrium contact angles by lithography and the UV nanoimprint technique. To consider the effect of the Cassie-Baxter and Wenzel states on the drainage properties, four different microperiodic structures were fabricated using the aspect ratio $f_{2} / f_{1}$ as the parameter. It appeared that the threshold level for hydrophobicity introduced by the microperiodic structures was below $90^{\circ}$ when the water droplet was positioned in the Cassie-Baxter state. In this case, the static wettability might correlate with the dynamic drainage properties. Throughout these evaluations, it was revealed that the sliding angle is more useful than the sliding velocity as an index of the drainage properties, when other conditions such as the height and volume of the water droplet were set appropriately.

A staggered pillar array was applied to the microperiodic structures in order to realize a highdrainage property. As the results, the sliding angle and sliding velocity reached $14^{\circ}$ and $171.9 \mathrm{~mm} / \mathrm{s}$, respectively, for a microperiodic structure with pitch $\tau=12 \mu \mathrm{m}$, aspect ratio $f_{2} / f_{1}=2$, depth $d=$ $5 \mu \mathrm{m}$, and equilibrium contact angle $\theta=103.9^{\circ}$, where a material with a sliding angle of $65^{\circ}$ on a smooth surface was used.

\section{Acknowledgments}

This research was supported in part by Grant No. 16H03166 from the Japan Society for the Promotion of Science, Visualization of Cancer by Cytokine Coding using Sensor Array with Photoresponsive Microcapsules (P.I.- M. Yamaguchi), Japan.

\section{References}

1 A. B. D. Cassie: Discuss. Faraday Soc. 3 (1948) 11.

2 R. N. Wenzel: Ind. Eng. Chem. 28 (1936) 988.

3 A. Solga, Z. Cerman, B. F. Striffler, M. Spaeth, and W. Barthlott: Bioinspiration Biomimetics 2 (2007) S126.

4 E. Ueda and P. A. Levkin: Adv. Mater. 25 (2013) 1234.

5 A. B. D. Cassie and S. Baxter: Trans. Faraday Soc. 40 (1944) 546.

6 H. K. Webb, R. J. Crawford, and E. P. Ivanova: Adv. Colloid. Interface. Sci. 210 (2014) 58.

7 B. Bhushan and Y. C. Jung: Prog. Mater. Sci. 56 (2011) 1.

8 C. Josserand and S. T. Thoroddsen: Annu. Rev. Fluid. Mech. 48 (2016) 365.

9 R. Fürstner, W. Barthlott, C. Neinhuis, and P. Walzel: Langmuir 21 (2005) 956.

10 A. Marmur: Langmuir 19 (2003) 8343.

11 E. Y. Bormashenko ed.: Wetting of Real Surfaces (De Gruyter GmbH, Berlin, 2013) Chap. 5. 
12 W. Chen, A. Y. Fadeev, M. C. Hsieh, D. Öner, J. Youngblood, and T. J. McCarthy: Langmuir 15 (1999) 3395.

13 M. Miwa, A. Nakajima, A. Fujishima, K. Hashimoto, and T. Watanabe: Langmuir 16 (2000) 5754.

14 K. Kurogi, H. Yanc, and K. Tsujii: Physicochem. Eng. Aspects 317 (2008) 592.

15 J. Seeger and F. Reifler: Text. Res. J. 79 (2009) 1565.

16 C. Lv, C. Yang, P. Hao, He. Feng, and O. Zheng: Langmuir 26 (2010) 8704.

17 M. Esashi: Jpn. J. Appl. Phys. 51 (2012) 080001-1.

18 M. J. Madou ed.: Fundamentals of Microfabrication (CRC Press, NewYork, 1997) 2nd ed., Chap. 1.

19 M. J. Madou ed.: Fundamentals of Microfabrication: The Science of Miniaturization (CRC Press, New York 2002) Chap. 1.

20 D. J. Resnick, W. J. Dauksher, D. Mancini, K. J. NordquistImprint, T. C. Bailey, S. Johnson, N. Stacey, J. G. Ekerdt, C. G. Willson, S. V. Sreenivasan, and N. Schumaker: J. Vac. Sci. Technol. B 21 (2003) 2624.

21 M. Nosonovsky and B. Bhushan eds.: Green Tribology, Green Energy and Technology (Springer-Verlag, Berlin, 2012) Chap. 2

22 E. Bormashenko: Colloid Polym Sci. 291 (2013) 339.

23 C. G. L. Furmige: J. Colloid Interface Sci. 17 (1962) 309.

24 Ch. Extrand and Y. Kumagai: J. Colloid Interface Sci. 170 (1995) 515.

25 J. Bico, U. Thiele, and D. Quéré: Colloids Surf. A. 206 (2002) 41.

26 N. Verplanck, Y. Coffinier, V. Thomy, and R. Boukherroub: Nanoscale Res. Lett. 2 (2007) 577.

27 N. Gupta, S. Sasikala, D. B. Mahadik, A. V. Rao, and H. C. Barshilia: Appl. Surf. Sci. 258 (2012) 9723.

28 A. Nakajima, S. Suzuki, Y. Kameshima, N. Yoshida, T. Watanabe, and K. Okada: Chem. Lett. 32 (2003) 1148.

29 E. Pierce, F. J. Carmona, and A. Amirfazli: Physicochem. Eng. Aspects 323 (2008) 73.

\section{About the Authors}

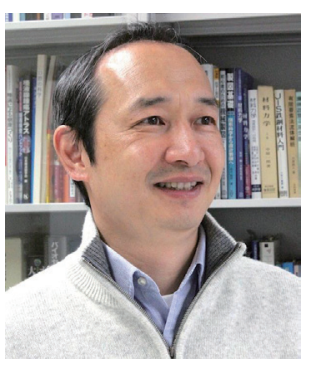

Masaki Yamaguchi received his B.S., M.S., and Ph.D. degrees in engineering from Shinshu University, Japan, in 1985, 1987, and 1994, respectively. He started as an assistant professor at Tokyo University of Agriculture and Technology, Japan, in 1995. He was an associate professor at the University of Toyama, Japan, from 1999 till 2008. He was a professor at Iwate University, Japan, from 2008 till 2015, when he moved to Shinshu University, where he is currently a professor of the Graduate School of Science \& Technology.

In 2001, he was given the Nikkei BP Technology Award in Medicine/Biotechnology Section by Nikkei Business Publications, Inc. for his achievements in the study of the noninvasive measurment of blood glucose via gingival crevicular fluid. In 2008, he was awarded the Product Prize with Nipro Corporation by the Society of Life Support Technology, Japan, for the development of a noninvasive salivary amylase monitor for human stress evaluation.

He coauthored 27 books, 114 refereed scientific and technical papers and 3 US patents concerning electromagnetic motors, medical sensors, robotics and their applications. His research interests focus on the development of medical biosensors and the improvement of surface wettability by applying three-dimensional periodic structures. He is a member of the IEEE EMBS (senior member), the Institute of Electrical Engineers of Japan (IEEJ, senior member), the Japanese Society for Medical and Biological Engineering (JSMBE), and the Japan Society of Mechanical Engineers (JSME).

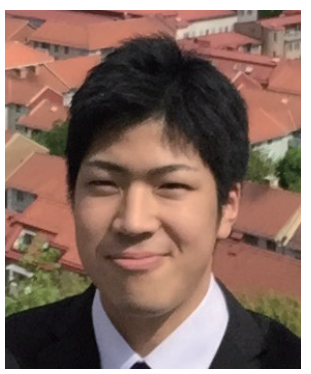

Tetsuhiro Sakata was born in Chiryu, Japan, in 1994. He received his B.S. degree from the Faculty of Textile Science and Technology of Shinshu University, Japan, in 2016. Currently, he is a master's course graduate student at Shinshu University. His research interests are on the numerical analysis of droplet behavior and wettability control by fine structures. 


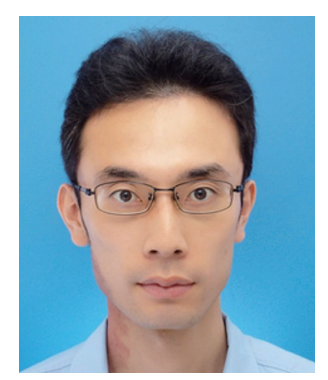

Tsuyoshi Chiba received his B.S. and M.S. degrees from the University of Tokyo in 2004 and 2006, respectively. He is currently in the doctoral program of Shinshu University. His research interests focus on the analysis of tactile sense by viscoelasticity and wettability. 